\title{
openheart Impact of transcatheter aortic valve implantation on mechanical dispersion
}

\author{
Lars Gunnar Klaeboe (10 , ${ }^{1,2}$ Pål Haugar Brekke, ${ }^{1}$ Lars Aaberge, ${ }^{1}$ Kristina Haugaa, ${ }^{1,2}$ \\ Thor Edvardsen ${ }^{1,2}$
}

\begin{abstract}
- Additional material is published online only. To view please visit the journal online (http://dx.doi.org/10.1136/ openhrt-2019-001199).
\end{abstract}

To cite: Klaeboe LG, Brekke PH, Aaberge L, et al. Impact of transcatheter aortic valve implantation on mechanical dispersion. Open Heart 2020;7:e001199. doi:10.1136/ openhrt-2019-001199

Received 30 November 2019 Revised 21 January 2020 Accepted 6 February 2020

\section{Check for updates}

(c) Author(s) (or their employer(s)) 2020. Re-use permitted under CC BY-NC. No commercial re-use. See rights and permissions. Published by BMJ.

${ }^{1}$ Department of Cardiology and Center for Cardiological Innovation, Oslo University Hospital Rikshospitalet, 0slo, Norway

${ }^{2}$ K.G. Jebsen Center for Cardiac Research, Institute of Clinical Medicine, Faculty of Medicine, University of Oslo, Oslo, Norway

Correspondence to Dr Thor Edvardsen; thor. edvardsen@medisin.uio.no

\section{ABSTRACT}

Objectives The physiological determinants of left ventricular (LV) mechanical dispersion (MD) are not fully explored. We aimed to investigate the impact of afterload reduction and changes in ventricular conduction on LV MD after transcatheter aortic valve implantation (TAVI). Methods Patients with severe aortic stenosis (AS) were examined in a prospective, repeated measures observational cohort study before and after an uncomplicated transfemoral TAVI in a single tertiary centre. LV MD was assessed by speckle tracking echocardiography. Valvulo-arterial impedance (ZVA) was used as a measure of global afterload.

Results We included 140 consecutive patients $(83 \pm 8$ years old, 49\% women, logistic EuroSCORE 16 \pm 10 ) with severe AS (valve area $0.7 \pm 0.2 \mathrm{~cm}^{2}$, mean transvalvular gradient $54 \pm 18 \mathrm{~mm} \mathrm{Hg}$ ) and a relatively preserved LV ejection fraction $(52 \% \pm 11 \%)$. After TAVI, we observed favourable changes in transvalvular gradients and ZVA in all patients. Compared with baseline, postprocedural MD was significantly lower in 108 patients with unchanged ventricular conduction $(55 \pm 17 \mathrm{~ms}$ vs $51 \pm 17 \mathrm{~ms}, \mathrm{p}=0.02)$ and higher in 28 patients with TAVI-induced left bundle branch block ( $51 \pm 13 \mathrm{~ms}$ vs $62 \pm 19 \mathrm{~ms}, \mathrm{p} \leq 0.001)$. During 22 \pm 9 months observation, 22 patients died. Postprocedural MD was associated with mortality in a univariate Cox regression model $(\mathrm{HR}=1.24$ (1.01-1.52), $p<0.04$, per 10 ms increase).

Conclusions Isolated afterload reduction was associated with reduction of MD, while concomitant impairment of ventricular conduction resulted in a more pronounced MD after TAVI, indicating that loading conditions and conduction should be considered when evaluating MD. A pronounced postprocedural LV MD was associated with mortality.

\section{INTRODUCTION}

The pressure overload in aortic stenosis (AS) leads to cardiac remodelling, myocardial fibrosis and ultimately impaired myocardial performance. ${ }^{1}$ While left ventricular (LV) ejection fraction $(\mathrm{EF})$ remains preserved until later stages of the disease, the reliability and prognostic value of global longitudinal strain (GLS) suggests that GLS may be a better marker of early systolic dysfunction in AS phenotypes with small cavity size and increased LV mass. ${ }^{1-4}$ Moreover, by studying

\section{Key questions}

What is already known about this subject?

- Knowledge regarding physiological determinants of mechanical dispersion in aortic stenosis is emerging, but the impact of changes in afterload and ventricular conduction on mechanical dispersion is previously unexplored.

What does this study add?

- The study adds information regarding changes in mechanical dispersion related to transcatheter aortic valve replacement. Mechanical dispersion appears to be a reproducible and robust measure of contractile heterogeneity under different loading conditions.

How might this impact on clinical practice?

- The current study indicates that prognostic information may be retained from mechanical dispersion after transcatheter aortic valve implantation.

the time course of segmental strain, subtle ventricular heterogeneity can be assessed by mechanical dispersion, which has been previously associated with adverse outcome, mainly ventricular arrhythmias, across a wide range of cardiac conditions..$^{5-10}$

Knowledge regarding the physiological determinants of mechanical dispersion in AS is emerging. Previously, older age, lower LVEF, larger LV mass and AS severity have been associated with increasing mechanical dispersion in AS. ${ }^{11}$ Whether afterload influences heterogeneity of ventricular contractions, however, is subject to discussion. ${ }^{12}$ Transcatheter aortic valve implantation (TAVI) causes abrupt haemodynamic changes and provides an opportunity to explore whether significant afterload reduction influences mechanical dyssynchrony. Furthermore, patients undergoing TAVI frequently acquire new-onset left bundle branch block (LBBB), enabling investigation of the separate and combined effects of changes in afterload and ventricular conduction on mechanical dispersion.

The primary aim of this study was to explore whether afterload and ventricular conduction 
are physiological determinants of LV dyssynchrony by assessing the immediate effect of TAVI on LV mechanical dispersion. Although such an experiment would require a selected group of patients without vascular TAVI complications that might affect loading conditions or require pacemakers, we wanted to evaluate whether prognostic information could be retained from mechanical dispersion after an uncomplicated procedure, since previous studies have linked pre-interventional mechanical dispersion to adverse outcome in AS. ${ }^{1113}$

\section{METHODS}

\section{Study population}

This prospective observational study was performed in the same patient cohort in which we have previously explored the contraction pattern of TAVI-induced LBBB. ${ }^{14}$ Consecutive patients with severe AS referred to a single tertiary centre (Oslo University Hospital Rikshospitalet, Oslo, Norway) undergoing uncomplicated TAVI between 1 January 2015 and 15 June 2017 were included in the study as previously described. ${ }^{13}$ Any postprocedural clinical follow-up was performed at referring hospitals and data were thus not available. However, all-cause mortality data were obtained from the Norwegian National Registry 15 June 2018 to ensure minimum 1-year observation. To restrict external factors that could have an impact on strain parameters, afterload and ventricular conduction, patients were not included if ventricular paced rhythm, paroxysmal arrhythmia or bigeminal ventricular ectopy were present at the time of either baseline or postoperative echocardiography, or if intravenous vasoactive drugs were administered. Moreover, patients who suffered stroke, vascular complications, cardiac tamponade, periprocedural myocardial infarction or bleeding leading to transfusion of two or more units of whole blood or red cells were excluded from the analysis (figure 1). Calculation of logistic EuroSCORE ${ }^{15}$ and New York Heart Association functional class was based on information obtained from medical records. Patients with previous myocardial infarction, a significant stenosis on an epicardial coronary artery, or a bypass graft $(\geq 50 \%$ diameter stenosis in the absence of collateral perfusion) on the coronary angiogram performed prior to TAVI were classified as having a history of coronary artery disease (CAD).

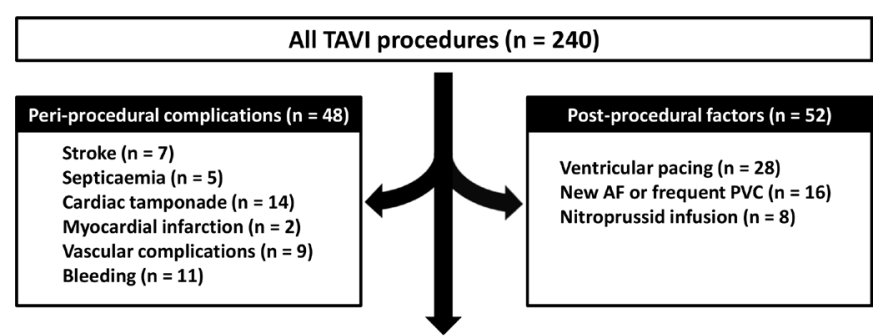

Uncomplicated TAVI procedures included in final analysis $(n=140)$

Figure 1 Flow chart of included patients. AF, atrial fibrillation; PVC, premature ventricular beats; TAVI, transcatheter aortic valve implantation.
Twelve-lead ECG was obtained at the time of the baseline and postoperative echocardiogram and confirmed at discharge. Right bundle branch block (RBBB) was defined as QRS duration $\geq 120$ ms with positive terminal deflection in V1. LBBB was defined according to strict ECG criteria. ${ }^{16}$ A QRS duration $<120 \mathrm{~ms}$ was classified as normal, while non-specific intraventricular conduction delay was defined as the presence of QRS duration $\geq 120$ ms not fulfilling the criteria of LBBB or RBBB. Informed consent was obtained from all study participants. There was no involvement by patients or patient organisations in the design or conduct of the present research project.

\section{Echocardiography}

Echocardiography was performed by experienced operators from the Section of Cardiac ultrasound, Department of Cardiology, Oslo University Hospital Rikshospitalet, using the Vivid E9 ultrasound system (GE Healthcare, Horten, Norway). Baseline echocardiography was performed at an initial evaluation visit. TAVI procedures were scheduled $112 \pm 67$ days after initial evaluation. Postprocedural echocardiography was performed before hospital discharge (in $96 \%$ on day 1 or 2 postprocedure). Echocardiographic analysis was performed offline and blinded with regards to clinical data using EchoPac V.201 (GE Healthcare). LV EF was calculated by the modified Simpson's method of discs. LV mass indexed for body surface area (BSA) was calculated by two-dimensional linear model using the Devereux formula. We obtained e' by tissue Doppler imaging in the septal annulus and E wave from mitral blood flow. Left atrial (LA) volume indexed for BSA was calculated by the biplane area-length method. The tricuspid annular plane systolic excursion was recorded from the focused right ventricular (RV) apical view. ${ }^{17}$ Valvulo-arterial impedance (ZVA), the sum of systolic arterial pressure and mean aortic transvalvular pressure gradient divided by stroke volume index, was assessed to estimate global afterload. ${ }^{18}$ GLS assessed by speckle tracking echocardiography was defined as the average peak longitudinal strain in a 16-segment LV model. $\mathrm{LV}$ mechanical dispersion defined as the SD of time from $\mathrm{Q} / \mathrm{R}$ on ECG to peak negative strain was assessed in 16 LV segments ${ }^{7}$ (figure 2). Multi-beat loops were stored in patients with atrial fibrillation and the average values of three loops from apical long-axis view and four and twochamber views with RR intervals compatible with built-in software constraints for calculating longitudinal strain were reported. A random sample of $20 \%$ of preprocedure echocardiography studies were re-analysed with speckle tracking to calculate intra-observer and inter-observer reproducibility in mechanical dispersion.

\section{TAVI procedure}

Transfemoral access was used in all TAVI procedures while patients were in conscious sedation. Deployed bioprosthesis were the balloon-expanded Edwards-Sapien 3 (Edwards Lifesciences, Orange County, California, USA; 121 patients) and Edwards-Sapien XT (1 patient) valves 


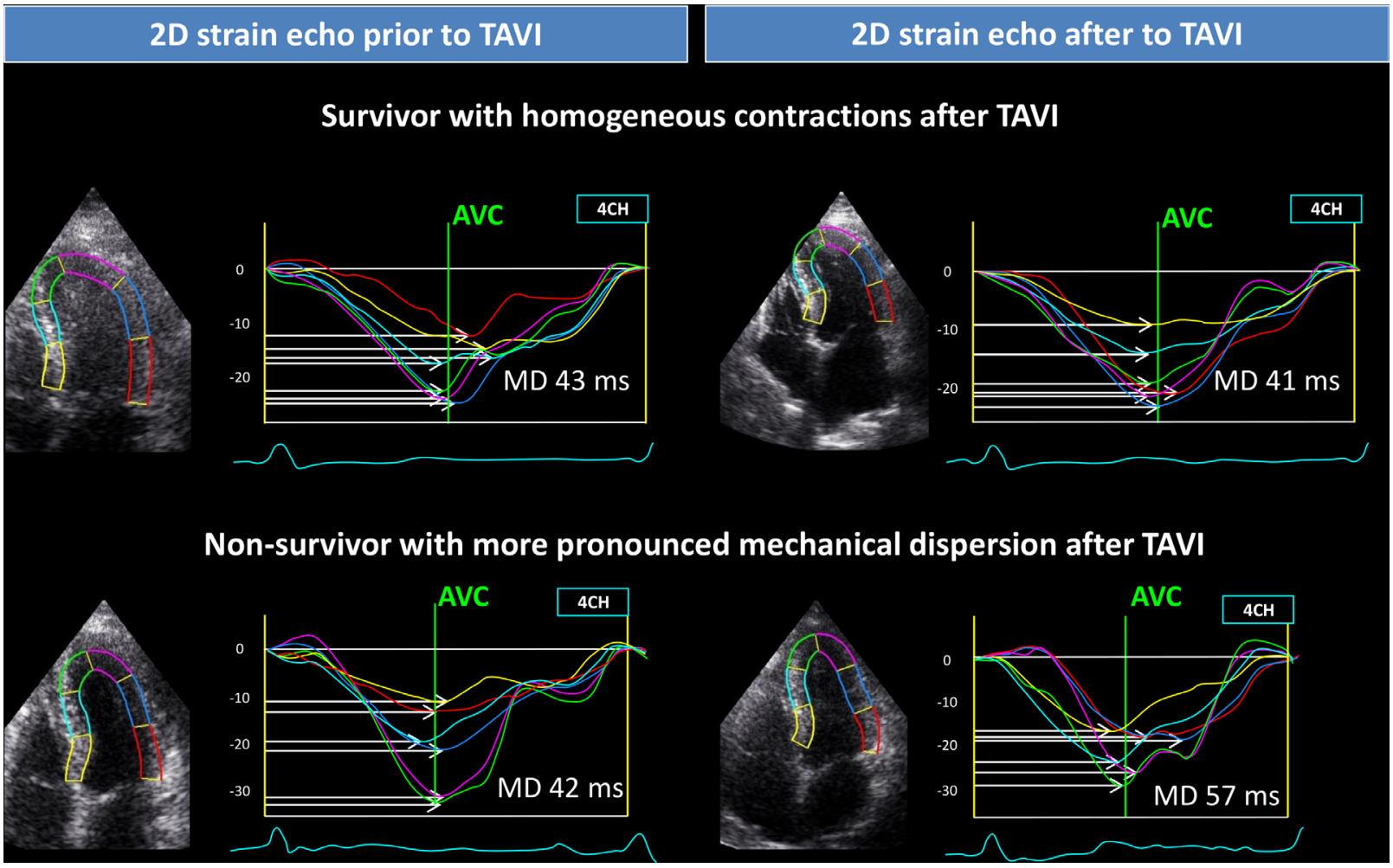

Figure 2 Speckle tracking echocardiography showing longitudinal strain curves in apical four-chamber view before and after TAVI in a survivor (upper panel) and non-survivor (lower panel) with unchanged ventricular conduction. White horizontal arrows indicate contraction duration, defined as time from Q/R on ECG to peak negative longitudinal strain. Mechanical dispersion was defined as the standard deviation of contraction duration from 16 left ventricular segments. The upper panel demonstrates a more homogeneous contraction pattern (lower mechanical dispersion) in a survivor before and after TAVI, whereas in the nonsurvivor (lower panel) postprocedural mechanical dispersion is more pronounced. AVC, aortic valve closure; MD, mechanical dispersion; TAVI, transcatheter aortic valve implantation.

and the self-expanding CoreValve Evolute R (Medtronic, Minneapolis, Minnesota, USA; 8 patients) and Symetis Acurate TF (Symetis SA, Ecublens, Switzerland; 10 patients) valves. Rapid RV pacing at $180 /$ min was used under deployment of balloon-expandable bioprosthesis, while self-expandable bioprosthesis was deployed without pacing or only under slow-rapid RV pacing at 120/min. After baseline echocardiography prior to TAVI, percutaneous coronary revascularisation (PCI) was performed in 25 patients with significant coronary artery stenosis. More men $(n=18)$ than women $(n=7)$ underwent staged PCI prior to TAVI $(\mathrm{p}=0.02)$. Twenty-eight patients were excluded due to ventricular pacing at the time of the postprocedural echocardiographic examination. Of these, 9 patients were paced transiently, while 19 received permanent pacemaker due to atrio-ventricular block. Postprocedural severe paravalvular regurgitation was not observed in any patients, while 133 patients $(95 \%)$ had less than moderate paravalvular regurgitation after the procedure.

\section{Statistics}

Data were presented as mean $\pm \mathrm{SD}$ or frequencies with percentages and compared by Student's t-test, paired Student's t-test, $\chi^{2}$ or Fischer's exact test as appropriate (SPSS Statistics V.22.0, SPSS). Linear associations were evaluated by linear regression or bivariate Pearson correlation. Intra-rater and inter-rater reproducibility was evaluated by intraclass correlation and Bland-Altman plots. Prognostic significance of mechanical dispersion was explored by Cox regression analysis. Kaplan-Meyer survival curves for postprocedural mechanical dispersion was created by using a $62 \mathrm{~ms}$ cut-off previously applied by Prihadi et $a l .{ }^{11}$ Two-sided $\mathrm{p}$ values $<0.05$ were considered statistically significant.

\section{RESULTS \\ Baseline clinical characteristics and echocardiography}

TAVI was performed in 240 consecutive patients. After excluding patients with procedural complications or peri-procedural factors that would influence afterload and ventricular conduction, a total of 140 patients $(83 \pm 8$ years old, $49 \%$ women) undergoing an uncomplicated TAVI procedure were included in the final analysis (figure 1). These patients had severe AS, mildly increased LV mass, preserved LVEF and signs of diastolic function with increased $\mathrm{E} / \mathrm{e}^{\prime}$, LA dilatation and mild pulmonary hypertension (table 1) prior to TAVI. (Baseline clinical characteristics and echocardiography in all patients and stratified according toventricular conduction are shown in online supplementary table 1 ) 
Table 1 Baseline clinical characteristics, ECG and echocardiography in complete cohort $(n=140)$

Baseline clinical characteristics

\begin{tabular}{|c|c|}
\hline Age & $83 \pm 7$ \\
\hline Blood pressure, diastolic, mm Hg & $73 \pm 11$ \\
\hline Blood pressure, systolic, $\mathrm{mm} \mathrm{Hg}$ & $140 \pm 24$ \\
\hline Body surface area, m² & $1.8 \pm 0.2$ \\
\hline Body mass index, $\mathrm{kg} / \mathrm{m}^{2}$ & $24.6 \pm 4.3$ \\
\hline CAD history, $\mathrm{n}(\%)$ & $73(52)$ \\
\hline Logistic EuroSCORE & $16.2 \pm 10.2$ \\
\hline Male/female, $\mathrm{n}$ (\% female) & $72 / 68(49)$ \\
\hline NYHA class & $2.5 \pm 0.6$ \\
\hline \multicolumn{2}{|l|}{ ECG } \\
\hline Sinus rhythm, $\mathrm{n}(\%)$ & $108(77)$ \\
\hline Atrial fibrillation, $\mathrm{n}(\%)$ & $32(23)$ \\
\hline QRS duration $<120 \mathrm{~ms}, \mathrm{n}(\%)$ & $113(81)$ \\
\hline Pre-existing conduction abnormalities, n (\%) & $27(19)$ \\
\hline LBBB, $n$ & 10 \\
\hline RBBB, $n$ & 9 \\
\hline Unspecific conduction abnormalities, $\mathrm{n}$ & 8 \\
\hline \multicolumn{2}{|l|}{ Echocardiography } \\
\hline Aortic valve area, $\mathrm{cm}^{2}$ & $0.7 \pm 0.2$ \\
\hline Aortic valve mean pressure gradient, $\mathrm{mm} \mathrm{Hg}$ & $54 \pm 18$ \\
\hline Aortic valve peak velocity, $\mathrm{m} / \mathrm{s}$ & $4.5 \pm 0.7$ \\
\hline $\mathrm{E} / \mathrm{e}^{\prime}$ & $21 \pm 11$ \\
\hline $\mathrm{LA}$ volume, $\mathrm{mL} / \mathrm{m}^{2}$ & $57 \pm 18$ \\
\hline LV end-diastolic diameter, mm & $50 \pm 8$ \\
\hline LV ejection fraction, \% & $52 \pm 11$ \\
\hline LV mass (men), g/cm² & $111 \pm 24$ \\
\hline LV mass (women), $\mathrm{g} / \mathrm{cm}^{2}$ & $102 \pm 22$ \\
\hline Stroke volume index, $\mathrm{mL} / \mathrm{m}^{2}$ & $40 \pm 10$ \\
\hline Tricuspid annular plane systolic excursion, mm & $21 \pm 6$ \\
\hline Tricuspid regurgitation gradient, $\mathrm{mm} \mathrm{Hg}$ & $36 \pm 13$ \\
\hline
\end{tabular}

Values are mean \pm SD deviation unless otherwise indicated. CAD, coronary artery disease; $e^{\prime}$, early diastolic mitral annular tissue velocity; $E$, early diastolic mitral inflow velocity; $L A$, left atrial; LBBB, left bundle branch block; LV, left ventricular; NYHA, New York Heart Association; RBBB, right bundle branch block.

Mean value of mechanical dispersion before TAVI was $54 \pm 19 \mathrm{~ms}$ in the total population. Patients with normal QRS duration before TAVI $(\mathrm{n}=113)$ had lower mechanical dispersion $(53 \pm 16 \mathrm{~ms}$ vs $60 \pm 20 \mathrm{~ms}, \mathrm{p}=0.05)$ than patients with pre-existing conduction abnormalities $(n=27)$.

Women were older $(84 \pm 5$ years vs $81 \pm 8$ years, $\mathrm{p}=0.02)$ and had lower prevalence of CAD $(31 \%$ vs $71 \%, \mathrm{p}<0.001)$ than men. Mean aortic gradient was higher $(60 \pm 18 \mathrm{~mm}$ $\mathrm{Hg}$ vs $48 \pm 15 \mathrm{~mm} \mathrm{Hg}, \mathrm{p}<0.001)$ and aortic valve area was lower $\left(0.6 \pm 0.1 \mathrm{~cm}^{2}\right.$ vs $\left.0.7 \pm 0.2 \mathrm{~cm}^{2}, \mathrm{p}<0.001\right)$ in women. Women also had better LVEF ( $55 \pm 8$ vs $49 \% \pm 12 \%$, $\mathrm{p}<0.001)$. There was, however, no gender difference in
GLS ( $-14.5 \% \pm 4.8 \%$ (men) vs $-15.7 \% \pm 3.8 \%$ (women), $\mathrm{p}=0.12)$ and mechanical dispersion $(52 \pm 17 \mathrm{~ms}$ (men) vs $55 \pm 16 \mathrm{~ms}$ (women), $\mathrm{p}=0.30]$ ) at baseline. Patients with $\mathrm{CAD}$ did not have significantly higher mechanical dispersion than patients without CAD ( $55 \pm 16 \mathrm{~ms}$ vs $52 \pm 17 \mathrm{~ms}$, $\mathrm{p}=0.42)$.

\section{TAVI-related changes in mechanical dispersion, ventricular conduction and afterload}

We observed a different response in mechanical dispersion after TAVI dependent on postprocedural conduction status. A decrease in mechanical dispersion was observed ( $55 \pm 17 \mathrm{~ms}$ vs $51 \pm 17 \mathrm{~ms}, \mathrm{p}=0.02)$ in 108 patients with unchanged conduction after TAVI, including 81 patients who maintained normal ventricular conduction and 27 patients with pre-existing persistent conduction abnormalities.

In order to further isolate the effect of afterload reduction, we performed a sensitivity analysis, excluding 25 patients who underwent staged PCI between baseline echocardiography and TAVI procedures, leaving the patients without the need for revascularisation who maintained a normal QRS duration $(\mathrm{n}=66)$ after TAVI. In this subgroup of patients, we found a postprocedural decrease in mechanical dispersion ( $54 \pm 17 \mathrm{~ms}$ vs $49 \pm 16 \mathrm{~ms}, \mathrm{p}=0.02)$.

Mechanical dispersion increased $(51 \pm 13 \mathrm{~ms}$ vs $62 \pm 19 \mathrm{~ms}$, $\mathrm{p}<0.001)$ in 28 patients with TAVI-induced LBBB. There were not enough patients to conclude on TAVI-induced changes regarding mechanical dispersion in new-onset $\operatorname{RBBB}(\mathrm{n}=2)$ and new-onset non-specific conduction disorder $(n=2)$.

For the whole cohort $(n=140)$, a weak, positive relationship was found between postprocedural change in mechanical dispersion and change in QRS duration $(\mathrm{R}=0.35, \mathrm{p}<0.001$ ) (figure 3). Substantial reductions of transvalvular gradients and ZVA after TAVI were observed regardless of acquired LBBB or unaffected ventricular conduction (table 2). In bivariate linear regression, there was no linear association between mechanical dispersion and ZVA $(\mathrm{R}=0.02, \mathrm{p}=0.85)$ at baseline. There was,

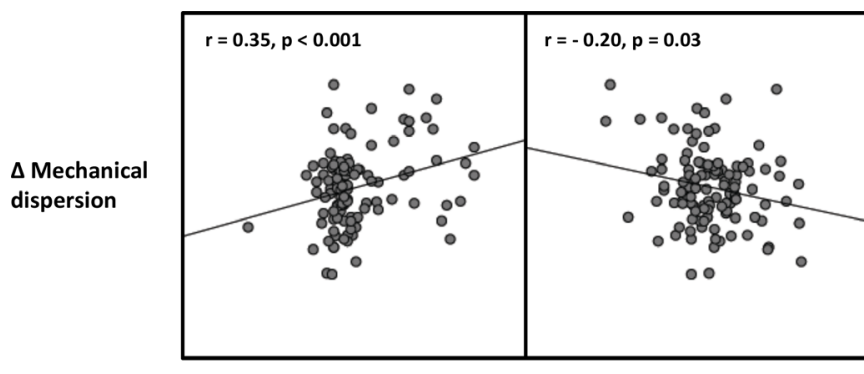

$\triangle$ QRS duration

$\Delta$ Valvulo-arterial impedance

Figure 3 Associations between change in ventricular conduction, global afterload and mechanical dispersion evaluated by bivariate Pearson correlation. A weak, positive relationship was found between change in QRS duration and change in mechanical dispersion (left panel). A weak, inverse relationship was found between change in mechanical dispersion and change in global afterload as expressed by valvulo-arterial impedance (right panel). 
Table 2 Myocardial function, afterload and ventricular conduction in patients with unchanged conduction status after TAVI $(n=108)$, new LBBB $(n=28)$, survivors $(n=118)$ and non-survivors $(n=22)$

\begin{tabular}{|c|c|c|c|}
\hline & Baseline & After TAVI & $P$ value \\
\hline \multicolumn{4}{|l|}{ Global longitudinal strain, \% } \\
\hline Unchanged conduction & $-15.3 \pm 4.4$ & $-16.5 \pm 3.9$ & $<0.001$ \\
\hline New LBBB & $-14.5 \pm 3.9$ & $-15.6 \pm 3.0$ & 0.03 \\
\hline Survivors & $-15.3 \pm 4.3$ & $-16.4 \pm 3.6$ & $<0.001$ \\
\hline Non-survivors & $-14.1 \pm 4.6$ & $-15.6 \pm 4.9$ & 0.04 \\
\hline \multicolumn{4}{|l|}{$\begin{array}{l}\text { Left ventricular ejection } \\
\text { fraction, } \%\end{array}$} \\
\hline Unchanged conduction & $53 \pm 11$ & $54 \pm 9$ & 0.17 \\
\hline New LBBB & $50 \pm 10$ & $52 \pm 8$ & 0.12 \\
\hline Survivors & $52 \pm 11$ & $54 \pm 9$ & $<0.001$ \\
\hline Non-survivors & $50 \pm 10$ & $51 \pm 10$ & 0.047 \\
\hline \multicolumn{4}{|l|}{ Mechanical dispersion, ms } \\
\hline Unchanged conduction & $55 \pm 17$ & $51 \pm 17$ & 0.023 \\
\hline New LBBB & $51 \pm 13$ & $62 \pm 19$ & $<0.001$ \\
\hline Survivors & $54 \pm 17$ & $53 \pm 18$ & 0.36 \\
\hline Non-survivors & $49 \pm 12$ & $60 \pm 18$ & 0.025 \\
\hline \multicolumn{4}{|l|}{$\begin{array}{l}\text { Aortic valve mean gradient, } \\
\mathrm{mm} \mathrm{Hg}\end{array}$} \\
\hline Unchanged conduction & $53 \pm 18$ & $13 \pm 5$ & $<0.001$ \\
\hline New LBBB & $59 \pm 15$ & $11 \pm 3$ & $<0.001$ \\
\hline Survivors & $54 \pm 19$ & $12 \pm 5$ & $<0.001$ \\
\hline Non-survivors & $49 \pm 13$ & $12 \pm 4$ & $<0.001$ \\
\hline
\end{tabular}

Valvulo-arterial impedance,

$\mathrm{mm} \mathrm{Hg} \mathrm{m} 2 / \mathrm{mL}$

\begin{tabular}{lrrr} 
Unchanged conduction & $5.1 \pm 1.1$ & $3.6 \pm 0.9$ & $<0.001$ \\
\hline New LBBB & $5.2 \pm 1.1$ & $3.4 \pm 0.8$ & $<0.001$ \\
\hline Survivors & $5.1 \pm 1.1$ & $3.6 \pm 0.9$ & $<0.001$ \\
\hline Non-survivors & $4.9 \pm 1.3$ & $3.2 \pm 0.8$ & $<0.001$ \\
QRS duration, ms & & & \\
Unchanged conduction & $106 \pm 23$ & $107 \pm 21$ & 0.19 \\
\hline New LBBB & $95 \pm 11$ & $145 \pm 13$ & $<0.001$ \\
Survivors & $103 \pm 22$ & $114 \pm 25$ & $<0.001$ \\
\hline Non-survivors & $105 \pm 20$ & $124 \pm 28$ & $<0.001$ \\
\hline
\end{tabular}

Values are mean \pm SD deviation.

LBBB, left bundle branch block; TAVI, transcatheter aortic valve implantation.

however, a weak, negative linear association between postprocedural change in mechanical dispersion and changes in ZVA ( $\mathrm{R}=-0.20$, $\mathrm{p}=0.03)$ (figure 3$)$.

\section{Intra-observer and inter-observer agreement}

For 28 patients $(20 \%)$ in whom baseline mechanical dispersion was re-evaluated, both intra-observer and inter-observer intraclass correlation were excellent at $0.95(95 \%$ CI 0.90 to $0.98, \mathrm{p}<0.001)$ and $0.91(95 \%$ CI
0.81 to 0.96 ), respectively. Bland-Altman plots (figure 4) and corresponding linear regression analyses revealed no sign of proportional bias.

\section{Exploratory survival analysis}

Twenty-two patients died during the $22 \pm 9$ months observation period. We observed an increase in mechanical dispersion after TAVI in 15 of 22 non-survivors. Mean values for mechanical dispersion before and after TAVI are shown in table 2.

In univariate survival analyses, postprocedural mechanical dispersion and increase in mechanical dispersion were the parameters significantly associated with increased mortality (table 3). Gender was not significantly associated with mortality in univariate Cox regression analysis (HR 1.80 (95\% CI 0.75 to 4.31 ), p=0.19). In separate bivariate models, an increase in mechanical dispersion of $10 \mathrm{~ms}$ compared with baseline $(\triangle \mathrm{MD})$ remained significantly associated with mortality when adjusted for all other variables shown in table 3 , while postprocedural mechanical dispersion remained significant when adjusted for CAD history, gender, LVEF, GLS, new-onset LBBB and change in QRS. Applying the same cut-off as Prihadi $e t$ $a l,{ }^{11}$ survival was significantly worse among patients with postprocedural mechanical dispersion $\geq 62 \mathrm{~ms}$ compared with patients with $\mathrm{LV}$ mechanical dispersion $<62 \mathrm{~ms}$ (log rank $p=0.02$, figure 5$)$. Restricting the analysis to patients without new-onset LBBB ( $\mathrm{n}=112)$ after TAVI, an increase in mechanical dispersion was significantly associated with mortality (univariate $\mathrm{HR}=1.43$ (95\% CI 1.08 to 1.88), $\mathrm{p}=0.013$, per $10 \mathrm{~ms}$ increase) .

\section{DISCUSSION}

The current study demonstrates TAVI-related changes in mechanical dispersion. While analytically isolating the effects of afterload reduction and alteration of conduction in an observational study is challenging, we observed a decrease in mechanical dispersion after TAVI procedures with unaffected electrical conduction. Conversely, TAVI-induced conduction delay was associated with an increase in mechanical dispersion. Elevated postprocedural mechanical dispersion was associated with higher mortality.

GLS has been shown to be inversely related to afterload, ${ }^{1920}$ while mechanical dispersion has been only weakly associated with LV load within the physiological range in hypertensive patients without hypertrophy or diastolic dysfunction. ${ }^{21}$ Following acute afterload relief, postprocedural mechanical dispersion was significantly lower than baseline values in patients in whom TAVI did not alter ventricular conduction. This aligns with the findings of Yamawaki et al, where speckle tracking imaging in 30 patients with severe AS and preserved LVEF showed improvement in regional mechanical dyssynchrony after surgical repair. ${ }^{22}$ The weak negative association with change in ZVA and the modest decrease in mechanical dispersion reported under non-physiological afterload 

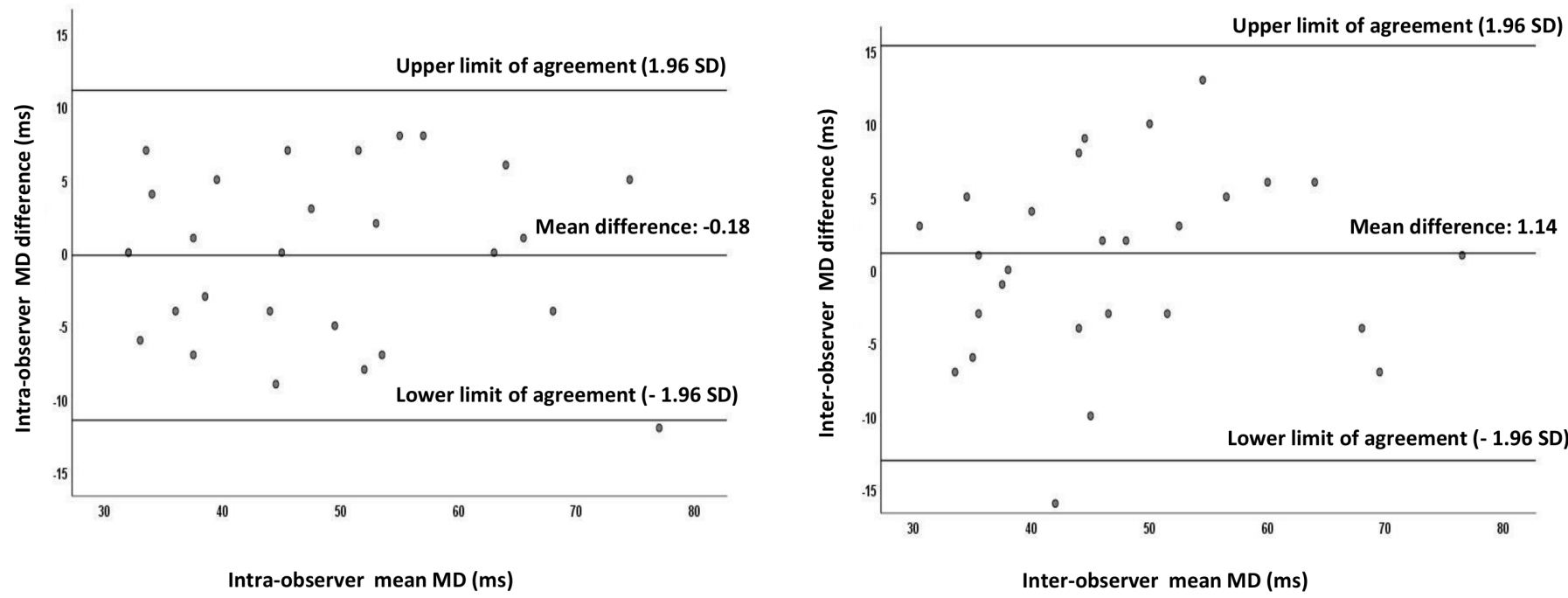

Figure 4 Intra-rater (left) and inter-rater (right) reliability evaluated by Bland-Altman plots. MD, mechanical dispersion.

decrease in this study may support the notion of mechanical dispersion as a deformation parameter relative insusceptible to changes in LV load within stable AS conditions.

In experimental animal studies evaluating the effects of aortic banding, both hypertrophic and fibrotic remodelling, as well as changes in afterload, have impact on longitudinal strain. ${ }^{23}$ Similarly, electrical propagation and
LBBB contraction patterns may be different in the remodelled myocardium in $\mathrm{AS} .{ }^{24}$ While we observed an increase in postprocedural mechanical dispersion in patients with TAVI-induced LBBB, the change was modest. The mean value of $62+19 \mathrm{~ms}$ was lower than mechanical dispersion in previous studies of LBBB patients, ${ }^{725}$ which was in fact not higher than the mechanical dispersion documented

Table 3 Cox regression analysis in 140 patients with severe AS undergoing an uncomplicated TAVI

\begin{tabular}{|c|c|c|c|c|c|c|}
\hline \multirow[b]{2}{*}{ Variables } & \multicolumn{2}{|l|}{ Univariate } & \multicolumn{2}{|c|}{$\begin{array}{l}\text { *Bivariate models with } \\
\text { adjusted postprocedural } \\
\text { MD }\end{array}$} & \multicolumn{2}{|c|}{$\begin{array}{l}\text { †Bivariate models with } \\
\text { adjusted } \triangle M D\end{array}$} \\
\hline & HR $(95 \% \mathrm{Cl})$ & $P$ value & HR $(95 \% \mathrm{Cl})$ & $P$ value & HR $(95 \%$ Cl) & $P$ value \\
\hline \multirow[t]{2}{*}{ CAD history } & 1.51 (0.63 to 3.62 ) & 0.35 & 1.47 (0.61 to 3.52 ) & 0.38 & 1.55 (0.65 to 3.71$)$ & 0.32 \\
\hline & & & $1.23(1.01 \text { to } 1.50)^{*}$ & 0.05 & $1.49(1.19$ to 1.88$) \dagger$ & 0.001 \\
\hline \multirow[t]{2}{*}{ Gender } & 1.80 (0.75 to 4.31$)$ & 0.19 & 1.84 (0.77 to 4.42$)$ & 0.17 & 1.65 (0.69 to 3.98$)$ & 0.27 \\
\hline & & & $1.23(1.01 \text { to } 1.49)^{*}$ & 0.04 & $1.47(1.17$ to 1.84$) \dagger$ & 0.001 \\
\hline \multirow[t]{2}{*}{ GLS per \% increase } & 1.03 (0.92 to 1.15$)$ & 0.67 & 0.99 (0.88 to 1.11$)$ & 0.88 & 0.99 (0.89 to 1.12$)$ & 0.97 \\
\hline & & & $1.24(1.00 \text { to } 1.55)^{*}$ & 0.05 & 1.50 (1.18 to 1.91$) \dagger$ & 0.001 \\
\hline \multirow[t]{2}{*}{ Logistic EuroSCORE } & 1.01 (0.97 to 1.05$)$ & 0.58 & 1.01 (0.97 to 1.05$)$ & 0.69 & 1.01 (0.97 to 1.05$)$ & 0.69 \\
\hline & & & $1.23(0.99 \text { to } 1.53)^{*}$ & 0.06 & $1.50(1.19$ to 1.89$) \dagger$ & 0.001 \\
\hline \multirow[t]{2}{*}{ LVEF per \% increase } & 0.98 (0.94 to 1.03$)$ & 0.41 & 0.99 (0.95 to 1.03$)$ & 0.57 & 0.99 (0.95 to 1.03$)$ & 0.53 \\
\hline & & & $1.23(1.00 \text { to } 1.51)^{*}$ & 0.05 & 1.49 (1.18 to 1.88$) \dagger$ & 0.001 \\
\hline MD per $10 \mathrm{~ms}$ increase & 1.23 (1.01 to 1.51$)$ & 0.04 & & & & \\
\hline$\Delta \mathrm{MD}$ per $10 \mathrm{~ms}$ increase & 1.50 (1.18 to 1.89$)$ & 0.001 & & & & \\
\hline \multirow[t]{2}{*}{ New LBBB (yes/no) } & 1.11 (0.41 to 3.01$)$ & 0.84 & 0.94 (0.34 to 2.57 ) & 0.90 & 0.61 (0.21 to 1.73 ) & 0.35 \\
\hline & & & $1.24(1.01 \text { to } 1.52)^{*}$ & 0.04 & $1.56(1.22$ to 1.99$) \dagger$ & $<0.001$ \\
\hline \multirow[t]{2}{*}{$\triangle Q R S$ duration, per $10 \mathrm{~ms}$ increase } & 1.01 (0.99 to 1.03$)$ & 0.20 & 1.10 (0.94 to 1.29$)$ & 0.24 & 1.02 (0.86 to 1.22$)$ & 0.80 \\
\hline & & & $1.28(1.03 \text { to } 1.59)^{*}$ & 0.03 & $1.58(1.23$ to 2.03$) \dagger$ & $<0.001$ \\
\hline \multirow[t]{2}{*}{ 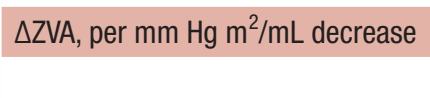 } & $1.04(0.72$ to 1.51$)$ & 0.83 & $1.12(0.76$ to 1.64$)$ & 0.58 & 1.24 (0.85 to 1.82$)$ & 0.26 \\
\hline & & & $1.20(0.94 \text { to } 1.53)^{*}$ & 0.14 & 1.55 (1.17 to 2.05$) \dagger$ & 0.002 \\
\hline
\end{tabular}

Rows marked with footnote symbols $\left(^{*}, \dagger\right)$ contain bivariate HR values for postprocedural MD and $\triangle M D$, respectively.

AS, aortic stenosis; CAD, coronary artery disease; GLS, global longitudinal strain; LBBB, left bundle branch block; LVEF, left ventricular ejection fraction; MD, mechanical dispersion; TAVI, transcatheter aortic valve implantation; ZVA, valvulo-arterial impedance. 


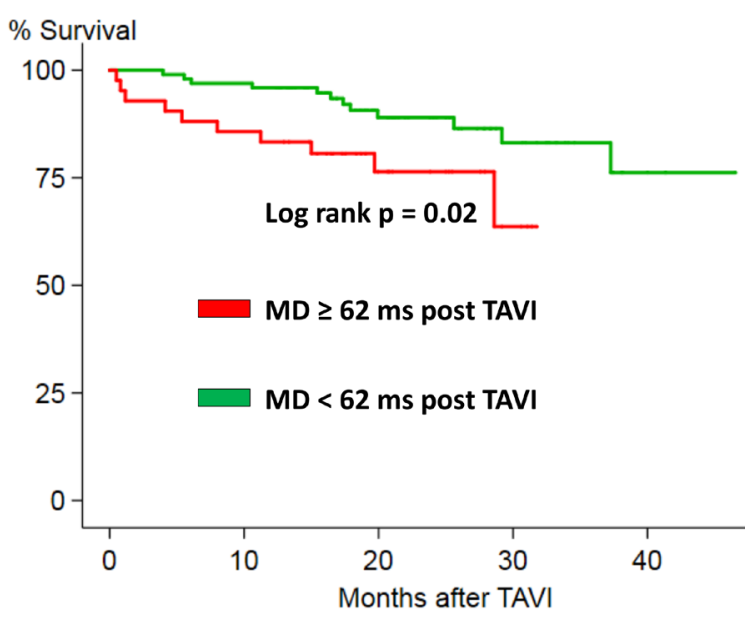

Number at risk

\begin{tabular}{|c|c|c|c|}
\hline $\mathrm{ms}$ & 98 & 95 & 53 \\
\hline $\mathrm{MD} \geq 62 \mathrm{~ms}$ & 42 & 36 & 17 \\
\hline
\end{tabular}

Figure 5 Kaplan-Meier survival curves according to mechanical dispersion after uncomplicated TAVI in 140 patients with aortic stenosis. Patients with postprocedural mechanical dispersion below $62 \mathrm{~ms}$ had better prognosis. $\mathrm{MD}$, mechanical dispersion; TAVI, transcatheter aortic valve implantation.

in a recent study of typical, unselected patients with severe AS $(71 \pm 24 \mathrm{~ms}) .{ }^{11}$ The moderate values of mechanical dispersion found in this subgroup harmonise well with our recent observation of a generally homogeneous contraction pattern in TAVI-induced LBBB early postprocedure. ${ }^{14}$ Although afterload relief seems to affect dyssynchrony to a lesser extent than new-onset LBBB early post-TAVI, LV adaption to sustained pressure overload may have altered myocardial sensitivity to changes in load and conduction. Whether similar alterations in LV load and conduction will affect other cardiac phenotypes differently should be elucidated further in order to optimise the information obtained from serial measurements and to differentiate test-retest variation from actual myocardial dysfunction.

The reproducibility of mechanical dispersion in the current study was excellent, with intraclass correlation coefficients within and between observers indicating that mechanical dispersion is a reliable tool suited for serial measurements in AS. However, further validation studies are needed to clarify inter-vendor and inter-software variability.

Mechanical dispersion has recently shown prognostic value in AS. ${ }^{1113}$ Our group has previously studied severe AS prior to surgical AVR, while Prihadi and colleagues were able to document prognostic value of mechanical dispersion also in moderate and mild AS. ${ }^{11}$ When correcting for age, AS severity and timing and occurrence of AVR, Prihadi et al found that mechanical dispersion had incremental prognostic value over LVEF. Performance of aortic valve intervention is decisive for outcome in severe symptomatic AS, but whether postprocedural prognostic information could be retained from mechanical dispersion has not been evaluated earlier. Indeed, the current study was conceived to elucidate physiological determinants of mechanical dispersion rather than prognostic factors related to TAVI, and sample size and number of events affect statistical power, precluding extensive survival modelling beyond exploratory analysis. Survival analysis results based on baseline data in this cohort would be conditional on an uncomplicated procedure and therefore subject to selection bias. Postprocedural mechanical dispersion assessed after an uncomplicated procedure, however, seems to represent a prognostic signal in our data, and it should not be affected by the selection bias of pre-interventional data.

In our study, neither QRS duration nor new-onset LBBB was statistically associated with adverse prognosis in multivariate analyses, but with a limited number of patients and events to base calculations on, these may be type II errors. As pointed out in a recent meta-analysis, bundle branch block will increase mechanical dispersion with an unknown effect of cardiac risk. ${ }^{26}$ The association between mechanical dispersion and mortality remained significant in a univariate Cox model restricted to 112 patients without TAVI-induced LBBB. While new-onset LBBB was related to an increase in mechanical dispersion, afterload reduction was associated with a decrease in mechanical dispersion in the remainder of patients. One possible interpretation is that the patients whose $\mathrm{LV}$ mechanics improved the most from TAVI-induced afterload reduction had the higher survival benefit. This may in turn be related to the degree of myocardial remodelling, ie, change in mechanical dispersion may reflect the degree to which AS-related remodelling is reversible. While there are other important differences between men and women undergoing TAVI, ${ }^{27}$ there were no gender differences regarding mechanical dispersion before and after intervention. The patients included in the present study were elderly with severe AS at high surgical risk and are likely to be representative of TAVI candidates worldwide. It is, however, an observational study with its inherent weaknesses. The study was primarily set up to investigate the procedure-associated change in speckle tracking parameters. To assess the effects of altered ventricular conduction and afterload, we analysed echocardiography examinations performed immediately after TAVI to avoid influence of reverse cardiac remodelling after removal of pathological pressure overload or change in medication that could alter loading conditions. However, afterload and strain parameters may have altered due to disease progression while awaiting intervention. The significant improvement of afterload, GLS and mechanical dispersion related to TAVI in the current study may, in fact, have been underestimated if worsening of transvalvular gradients and myocardial function were systematically occurring while patients were waiting for TAVI. Furthermore, it would have interesting to study postprocedural cardiac remodelling and ventricular conduction in more detail over time. Mid-term or long-term follow-up data were, however, not available in our study. 


\section{CONCLUSIONS}

Mechanical dispersion was associated with changes in ventricular conduction and afterload in patients treated for severe AS by TAVI. In comparisons of mechanical dispersion between groups, or in longitudinal follow-up studies, afterload and particularly ventricular conduction abnormalities should be taken into account. Despite the influence of these external factors, postprocedural mechanical dispersion remained associated with mortality, strengthening the interpretation of mechanical dispersion as a marker of inherent myocardial properties.

\section{Twitter Lars Gunnar Klaeboe @|klaboe}

Contributors LGK, KH and TE planned the design of this study. LGK collected and analysed echocardiographic data. LGK, PHB, LA, KH and TE contributed to data analysis and interpretation. LGK drafted the manuscript. All authors critically revised the manuscript and approved the final version.

Funding Center for Cardiological Innovation is supported by a grant (203489) from the Research Council of Norway and the K.G. Jebsen Center for Cardiac Research. This work was supported by the Research Council of Norway funding the Center for Cardiological Innovation (203489) and the K.G. Jebsen Center for Cardiac Research.

\section{Competing interests None declared.}

Patient consent for publication Not required.

Ethics approval The study was approved by the Regional Committee for Medical Research Ethics and complied with the Declaration of Helsinki.

Provenance and peer review Not commissioned; externally peer reviewed.

Data availability statement All data relevant to the study are included in the article.

Open access This is an open access article distributed in accordance with the Creative Commons Attribution Non Commercial (CC BY-NC 4.0) license, which permits others to distribute, remix, adapt, build upon this work non-commercially, and license their derivative works on different terms, provided the original work is properly cited, appropriate credit is given, any changes made indicated, and the use is non-commercial. See: http://creativecommons.org/licenses/by-nc/4.0/.

\section{ORCID iD}

Lars Gunnar Klaeboe http://orcid.org/0000-0002-9801-7905

\section{REFERENCES}

1 Dahl JS, Magne J, Pellikka PA, et al. Assessment of subclinical left ventricular dysfunction in aortic stenosis. JACC Cardiovasc Imaging 2019;12:163-71.

2 Baumgartner $\mathrm{H}$, Hung J, Bermejo J, et al. Recommendations on the echocardiographic assessment of aortic valve stenosis: a focused update from the European association of cardiovascular imaging and the American Society of echocardiography. J Am Soc Echocardiogr 2017;30:372-92.

3 Levy-Neuman S, Meledin V, Gandelman G, et al. The association between longitudinal strain at rest and stress and outcome in asymptomatic patients with moderate and severe aortic stenosis. $J$ Am Soc Echocardiogr 2019;32:722-9.

4 Magne J, Cosyns B, Popescu BA, et al. Distribution and prognostic significance of left ventricular global longitudinal strain in asymptomatic significant aortic stenosis: an individual participant data meta-analysis. JACC Cardiovasc Imaging 2019;12:84-92.

5 Barros MVL, Leren IS, Edvardsen T, et al. Mechanical dispersion assessed by strain echocardiography is associated with malignant arrhythmias in Chagas cardiomyopathy. J Am Soc Echocardiogr 2016;29:368-74.

6 Haland TF, Almaas VM, Hasselberg NE, et al. Strain echocardiography is related to fibrosis and ventricular arrhythmias in hypertrophic cardiomyopathy. Eur Heart J Cardiovasc Imaging 2016;17:613-21.

7 Haugaa KH, Goebel B, Dahlslett T, et al. Risk assessment of ventricular arrhythmias in patients with nonischemic dilated cardiomyopathy by strain echocardiography. J Am Soc Echocardiogr 2012;25:667-73.

8 Haugaa KH, Grenne BL, Eek CH, et al. Strain echocardiography improves risk prediction of ventricular arrhythmias after myocardial infarction. JACC Cardiovasc Imaging 2013;6:841-50.

9 Leren IS, Hasselberg NE, Saberniak J, et al. Cardiac mechanical alterations and genotype specific differences in subjects with long QT syndrome. JACC Cardiovasc Imaging 2015;8:501-10.

10 Perry R, Patil S, Marx C, et al. Advanced echocardiographic imaging for prediction of SCD in moderate and severe LV systolic function. $J$ Am Coll Cardiol Img.

11 Prihadi EA, Vollema EM, Ng ACT, et al. Determinants and prognostic implications of left ventricular mechanical dispersion in aortic stenosis. Eur Heart J Cardiovasc Imaging 2019;20:740-8.

12 Donal E, Galli E, Cosyns B. Twenty years after starting cardiac resynchronization therapy, do we understand the electromechanical coupling? Eur Heart J Cardiovasc Imaging 2019;20:257-9.

13 Klaeboe LG, Haland TF, Leren IS, et al. Prognostic value of left ventricular deformation parameters in patients with severe aortic stenosis: a pilot study of the usefulness of strain echocardiography. J Am Soc Echocardiogr 2017;30:727-35.

14 Klaeboe LG, Brekke PH, Lie Øyvind $\mathrm{H}$, et al. Classical mechanical dyssynchrony is rare in transcatheter aortic valve implantationinduced left bundle branch block. Eur Heart J Cardiovasc Imaging 2019;20:271-8.

15 Roques Fet al. The logistic EuroSCORE. Eur Heart J 2003;24:882-2.

16 Strauss DG, Selvester RH, Wagner GS. Defining left bundle branch block in the era of cardiac resynchronization therapy. Am J Cardiol 2011;107:927-34.

17 Lang RM, Badano LP, Mor-Avi V, et al. Recommendations for cardiac chamber quantification by echocardiography in adults: an update from the American Society of echocardiography and the European association of cardiovascular imaging. J Am Soc Echocardiogr 2015;28:1-39.

18 Briand M, Dumesnil JG, Kadem L, et al. Reduced systemic arterial compliance impacts significantly on left ventricular afterload and function in aortic stenosis. J Am Coll Cardiol 2005;46:291-8.

19 Carasso S, Cohen O, Mutlak D, et al. Differential effects of afterload on left ventricular long- and short-axis function: insights from a clinical model of patients with aortic valve stenosis undergoing aortic valve replacement. Am Heart J 2009;158:540-5.

20 Yotti R, Bermejo J, Benito Y, et al. Validation of noninvasive indices of global systolic function in patients with normal and abnormal loading conditions. A simultaneous echocardiography pressure-volume catheterization study. Circ Cardiovasc Imaging 2014;7:164-72.

21 Kosmala W, Przewlocka-Kosmala M, Sharman JE, et al. Stability of left ventricular longitudinal and circumferential deformation over time and standard loading conditions. Eur Heart J Cardiovasc Imaging 2017;18:1001-7.

22 Yamawaki K, Tanaka H, Matsumoto K, et al. Impact of left ventricular afterload on longitudinal dyssynchrony in patients with severe aortic stenosis and preserved ejection fraction. Circ J 2012;76:744-51.

23 Reant P, Metras A, Detaille D, et al. Impact of afterload increase on left ventricular myocardial deformation indices. J Am Soc Echocardiogr 2016;29:1217-28.

24 Auffret V, Martins RP, Daubert C, et al. Idiopathic/latrogenic left bundle branch block-induced reversible left ventricle dysfunction: jacc state-of-the-art review. J Am Coll Cardiol 2018;72:3177-88.

25 Stankovic I, Janicijevic A, Dimic A, et al. Mechanical dispersion is associated with poor outcome in heart failure with a severely depressed left ventricular function and bundle branch blocks. Ann Med 2018;50:128-38.

26 Kawakami H, Nerlekar N, Haugaa KH, et al. Prediction of ventricular arrhythmias with left ventricular mechanical dispersion: a systematic review and meta-analysis. JACC Cardiovasc Imaging 2020;13:562572.

27 D'Ascenzo F, Gonella A, Moretti C, et al. Gender differences in patients undergoing TAVI: a multicentre study. Eurolntervention 2013;9:367-72. 